\title{
New Exact Superposition Solutions to KdV2 Equation
}

\author{
Piotr Rozmej $\mathbb{D}^{1}$ and Anna Karczewska $\mathbb{D}^{2}$ \\ ${ }^{1}$ Faculty of Physics and Astronomy, University of Zielona Góra, Szafrana 4a, 65-246 Zielona Góra, Poland \\ ${ }^{2}$ Faculty of Mathematics, Computer Science and Econometrics, University of Zielona Góra, Szafrana 4a, 65-246 Zielona Góra, Poland
}

Correspondence should be addressed to Piotr Rozmej; p.rozmej@if.uz.zgora.pl

Received 27 September 2017; Revised 16 January 2018; Accepted 1 February 2018; Published 28 February 2018

Academic Editor: Antonio Scarfone

Copyright (C) 2018 Piotr Rozmej and Anna Karczewska. This is an open access article distributed under the Creative Commons Attribution License, which permits unrestricted use, distribution, and reproduction in any medium, provided the original work is properly cited.

\begin{abstract}
New exact solutions to the KdV2 equation (also known as the extended KdV equation) are constructed. The KdV2 equation is a second-order approximation of the set of Boussinesq's equations for shallow water waves which in first-order approximation yields $\mathrm{KdV}$. The exact solutions $(A / 2)\left(\mathrm{dn}^{2}[B(x-v t), m] \pm \sqrt{m} \mathrm{cn}[B(x-v t), m] \mathrm{dn}[B(x-v t), m]\right)+D$ in the form of periodic functions found in the paper complement other forms of exact solutions to KdV2 obtained earlier, that is, the solitonic ones and periodic ones given by single $\mathrm{cn}^{2}$ or $\mathrm{dn}^{2}$ Jacobi elliptic functions.
\end{abstract}

\section{Introduction}

Water waves have attracted the interest of scientists for at least two centuries. One hundred and seventy years ago Stokes [1] showed that waves described by nonlinear models can be periodic. In this way he pioneered the field of nonlinear hydrodynamics. The next important step was made by Boussinesq [2], though his achievement went unnoticed for many years. The most important approximation of the set of Euler's hydrodynamic equations was made by Korteweg and de Vries [3] who obtained a single nonlinear dispersive wave equation, called nowadays the $\mathrm{KdV}$ equation in their names. $\mathrm{KdV}$ became so famous since it constitutes a firstorder approximation for nonlinear waves in many fields: hydrodynamics, magnetohydrodynamics, electrodynamics, optics, and mathematical biology; see, for example, monographs [4-6]. It consists of the mathematically simplest terms representing the interplay of nonlinearity and dispersion. For some ranges of values of equation coefficients these two counteracting effects may cancel admitting solutions in the form of unidirectional waves of permanent shapes. $\mathrm{KdV}$ is integrable and possesses an infinite number of invariants. Its analytic solutions were found in the forms of single solitons, multisolitons, and periodic functions (cnoidal functions); see, for example, $[7,8]$ and monographs $[4-6,9-11]$.
For the shallow water problem leading to $\mathrm{KdV}$, two small parameters are assumed: wave amplitude/depth $\alpha=(A / H)$ and depth/wavelength squared $\beta=(H / L)^{2}$. Then the perturbation approach to Euler's equations for the irrotational motion of inviscid fluid is applied. Limitation to the terms of first order in $\alpha, \beta$ yields the $\mathrm{KdV}$ equation in the following form (expressed in scaled dimensionless variables in fixed reference frame):

$$
\eta_{t}+\eta_{x}+\frac{3}{2} \alpha \eta \eta_{x}+\frac{1}{6} \beta \eta_{3 x}=0
$$

Here and below the low indexes denote partial derivatives; for example, $\eta_{t} \equiv \partial \eta / \partial t$ and $\eta_{k x} \equiv \partial^{k} \eta / \partial x_{k}$.

One of reasons for the enormous success of the $\mathrm{KdV}$ equation is its simplicity and integrability. However, $\mathrm{KdV}$ is derived under the assumption that both $\alpha$ and $\beta$ parameters are small. Therefore one should not expect that KdV can properly describe shallow water waves for larger values of parameters $\alpha, \beta$. In principle, the extended $\mathrm{KdV}$ equation, obtained in second-order approximation with respect to these parameters, should be applicable for a wider range of parameters $\alpha, \beta$ than the $\mathrm{KdV}$ equation. 
The next, second-order approximation to Euler's equations for long waves over a shallow riverbed is

$$
\begin{aligned}
\eta_{t}+\eta_{x} & +\frac{3}{2} \alpha \eta \eta_{x}+\frac{1}{6} \beta \eta_{3 x}-\frac{3}{8} \alpha^{2} \eta^{2} \eta_{x} \\
& +\alpha \beta\left(\frac{23}{24} \eta_{x} \eta_{2 x}+\frac{5}{12} \eta \eta_{3 x}\right)+\frac{19}{360} \beta^{2} \eta_{5 x}=0
\end{aligned}
$$

This equation was first derived by Marchant and Smyth [12] and called the extended KdV. Later (2) was derived in a different way in [13] and as a by-product in derivation of the equation for waves over uneven bottom in $[14,15]$. Therefore exact solutions of (2) are the best initial conditions for performing numerical evolution of waves entering the regions where the bottom changes; see [16]. We call it $K d V 2$. $\mathrm{KdV} 2$ is not integrable. Contrary to $\mathrm{KdV}$ it has only one conservation law (mass or equivalent volume). On the other hand, as pointed out in [17], there exist adiabatic invariants of KdV2 in which relative deviations from constant values are very small (of the order of $0\left(\alpha^{3}\right)$ ).

Despite its nonintegrability, KdV2 possesses exact analytic solutions. The first class of such solutions, single solitonic solutions found by us, is published in [15]. These solutions have the same form sech ${ }^{2}$ as $\mathrm{KdV}$ solitonic solutions but slightly different coefficients. Following this discovery we came across papers by Khare and Saxena [18-20] who showed that there exist classes of nonlinear equations which possess exact solutions in the form of hyperbolic or Jacobi elliptic functions. They showed also that besides the usual solitonic $\left(\mathrm{sech}^{2}\right)$ and periodic cnoidal $\left(\mathrm{cn}^{2}\right)$ solutions other new solutions in the form of superpositions of hyperbolic or Jacobi elliptic functions exist. Since KdV belongs to these classes we formulated the hypothesis that this applied to KdV2, as well. In [21] this hypothesis was verified for periodic cnoidal $\left(\mathrm{cn}^{2}\right)$ solutions. Using an algebraic approach we showed there that for KdV2 (2) there exist analytic periodic solutions in the same form as $\mathrm{KdV}$ solutions; that is,

$$
\eta(x, t)=\mathrm{cn}^{2}[B(x-v t), m]+D .
$$

The formulas for the parameters of solutions $B, D, v, m$ were given explicitly as functions of the coefficients $\alpha, \beta$ of KdV2 (2). It appeared that KdV2 imposed some restrictions on the ranges of solution parameters when compared to KdV.

In [22], following Khare and Saxena [18], we checked our hypothesis for KdV2 solutions of the form

$$
\begin{aligned}
& \eta_{ \pm}(y)=\frac{A}{2}\left\{\operatorname{dn}^{2}[B(x-v t), m]\right. \\
& \pm \sqrt{m} \operatorname{cn}(B y, m) \operatorname{dn}[B(x-v t), m]\} .
\end{aligned}
$$

It was proved that both functions (4) satisfy (1); moreover explicit formulas for coefficients of (4) were given. However, this closed-form mathematical solution does not fulfil important physical condition that the mean fluid level remains the same for arbitrary wave.

This article complements $[15,21,22]$ by giving physically relevant solutions to $\mathrm{KdV} 2$ in the form of superpositions. In Section 2 equations determining the coefficients of the superposition solution are derived. The conditions necessary for the solution to describe shallow water waves are imposed on the solutions in Section 3 and formulas for the coefficients of the solution are obtained. In Section 4 some examples of solutions are presented with additional verification by numerical evolution of the obtained solutions according to (2). Section 5 contains conclusions.

Our idea to look for exact solutions to KdV2 in the same forms as solutions to $\mathrm{KdV}$ gained recently a strong support by results of Abraham-Shrauner [23].

1.1. Algebraic Approach to $K d V$. In order to present the approach we show the KdV case first. Assume solutions to $\mathrm{KdV}$ in the following form:

$$
\begin{aligned}
\eta_{ \pm}(y)= & \frac{A}{2}\left[\operatorname{dn}^{2}(B y, m) \pm \sqrt{m} \mathrm{cn}(B y, m) \operatorname{dn}(B y, m)\right] \\
& +D
\end{aligned}
$$

where $A, B, D, v$ are yet unknown constants ( $m$ is the elliptic parameter) which have the same meaning as in a single $\mathrm{cn}^{2}$ solution. Coefficient $D$ is necessary in order to maintain, for arbitrary $m$, the same volume for a wave's elevations and depressions with respect to the undisturbed water level.

Introduce $y:=x-v t$. Then $\eta(x, t)=\eta(y), \eta_{t}=-v \eta_{y}$ and (1) takes the form of an ODE:

$$
(1-v) \eta_{y}+\frac{3}{2} \alpha \eta \eta_{y}+\frac{1}{6} \beta \eta_{3 y}=0 .
$$

Insertion of (5) into (6) gives (common factor $\mathrm{CF}=$ $\left.A B \sqrt{m}(\sqrt{m} \mathrm{cn}+\mathrm{dn})^{2} \mathrm{sn}\right)$

$$
\mathrm{CF}\left(F_{0}+F_{2} \mathrm{cn}^{2}+F_{11} \mathrm{cn} \mathrm{dn}\right)=0 .
$$

Then there are three conditions on the solution

$$
\begin{aligned}
F_{0}= & 9 \alpha A-9 \alpha A m-2 \beta B^{2}+10 \beta B^{2} m+18 \alpha D-12 v \\
& +12=0, \\
F_{2}= & 9 \alpha A m-12 \beta B^{2} m=0, \\
F_{11}= & 9 \alpha A \sqrt{m}-12 \beta B^{2} \sqrt{m}=0 .
\end{aligned}
$$

Equations (9) and (10) are equivalent and yield

$$
B=\sqrt{\frac{3 \alpha}{4 \beta} A .}
$$

Insertion of this into (8) gives

$$
\alpha A(m-5)-4(3 \alpha D+2)+8 v=0 .
$$

Periodicity condition implies

$$
L=\frac{4 K(m)}{B}
$$

Then volume conservation condition determines $D$ as

$$
D=-\frac{A}{2} \frac{E(m)}{K(m)} .
$$


Finally insertion of $D$ into (12) gives velocity as

$$
v=1+\frac{\alpha A}{8}\left[5-m-6 \frac{E(m)}{K(m)}\right] \equiv 1+\frac{\alpha A}{8} E K(m) .
$$

$K(m)$ and $E(m)$ which appear in (13)-(15) are the complete elliptic integrals of the first kind and the second kind, respectively.

Equations (11), (14), and (15) express coefficients $B, D, v$ of the superposition solution (4) as functions of amplitude $A$, elliptic parameter $m \in[0,1]$, and parameters $\alpha, \beta$ of the $\mathrm{KdV}$ equation. In principle, these equations admit arbitrary amplitude of KdV solution in form (4).

Coefficients $B, D, v$ and the wavelength $L$ obtained above for solution (4) are different form coefficients of usual cnoidal solutions in form (3). In particular, since the function

$$
E K(m)=\left[5-m-6 \frac{E(m)}{K(m)}\right]
$$

changes its sign at $m \approx 0.449834$ the velocity dependence of wave (4) is much different than that of the $\mathrm{cn}^{2}$ wave [For $\mathrm{cn}^{2}$ wave $v=1+(\alpha A / 2 m)(2-m-3(E(m) / K(m)))]$; see (24) in [21]. Examples of $m$ dependence of velocity (15) are displayed in Figure 2.

\section{Algebraic Approach to KdV2}

Now, we look for solutions to KdV2 (2) in same form (5). In this case the corresponding ODE takes the form

$$
\begin{aligned}
& (1-v) \eta_{y}+\frac{3}{2} \alpha \eta \eta_{y}+\frac{1}{6} \beta \eta_{3 y}-\frac{3}{8} \alpha^{2} \eta^{2} \eta_{y} \\
& \quad+\alpha \beta\left(\frac{23}{24} \eta_{y} \eta_{2 y}+\frac{5}{12} \eta \eta_{3 y}\right)+\frac{19}{360} \beta^{2} \eta_{5 y}=0 .
\end{aligned}
$$

Assume solutions to KdV2 in form (5):

$$
\begin{aligned}
\eta_{ \pm}(y) & \\
= & \frac{A}{2}\left[\mathrm{dn}^{2}(B y, m) \pm \sqrt{m} \mathrm{cn}(B y, m) \mathrm{dn}(B y, m)\right] \\
& +D,
\end{aligned}
$$

where $A, B, D, v$ are yet unknown constants ( $m$ is the elliptic parameter) which have the same meaning as previously.

Insertion of (5) to (17) yields

$$
\mathrm{CF}\left(F_{0}+F_{2} \mathrm{cn}^{2}+F_{4} \mathrm{cn}^{4}+F_{11} \mathrm{cndn}+F_{31} \mathrm{cn}{ }^{3} \mathrm{dn}\right)=0,
$$

where common factor is

$$
\mathrm{CF}=A B \sqrt{m}(\sqrt{m} \mathrm{cn}+\mathrm{dn})^{2} \mathrm{sn} .
$$

Equation (19) is satisfied for arbitrary arguments when all coefficients $F_{0}, \ldots, F_{31}$ vanish simultaneously. This imposes five conditions on parameters:

$$
\begin{gathered}
F_{0}=135 \alpha^{2} A^{2}(m-1)^{2}+60 \alpha A(m-1) \\
\cdot\left(\beta B^{2}(48 m-5)-9 \alpha D+18\right)
\end{gathered}
$$

$$
\begin{aligned}
& -4\left[19 \beta^{2} B^{4}\left(61 m^{2}-46 m+1\right)\right. \\
& +30 \alpha D\left(5 \beta B^{2}(5 m-1)+18\right)+60 \beta B^{2}(5 m-1) \\
& \left.-135 \alpha^{2} D^{2}+360\right]+1440 v=0
\end{aligned}
$$

$$
\begin{aligned}
F_{2} & =-15 m\left[27 \alpha^{2} A^{2}(m-1)\right. \\
& -12 \alpha A\left(\beta B^{2}(37-59 m)+3 \alpha D-6\right) \\
& \left.-16 \beta B^{2}\left(38 \beta B^{2}(2 m-1)+15 \alpha D+6\right)\right]=0, \\
F_{4} & =90 m^{2}\left(3 \alpha^{2} A^{2}+86 \alpha A \beta B^{2}-152 \beta^{2} B^{4}\right)=0, \\
F_{11} & =-30 \sqrt{m}\left[9 \alpha^{2} A^{2}(m-1)\right. \\
& -3 \alpha A\left(\beta B^{2}(31-75 m)+6 \alpha D-12\right) \\
& \left.-4 \beta B^{2}\left(19 \beta B^{2}(5 m-1)+30 \alpha D+12\right)\right]=0, \\
F_{31} & =90 m^{3 / 2}\left(3 \alpha^{2} A^{2}+86 \alpha A \beta B^{2}-152 \beta^{2} B^{4}\right)=0 .
\end{aligned}
$$

As shown previously in [22] (23) and (25) are equivalent. Denote $z=\beta B^{2} / \alpha A$. Then roots of (25) are the same as those in the cases of solitonic [15] and $\mathrm{cn}^{2}$ solutions [21]; that is,

$$
z_{1 / 2}=\frac{43 \mp \sqrt{2305}}{152} .
$$

In principle we should discuss both cases.

Express (21), (22), and (24) through $z$ by substituting

$$
B=\sqrt{\frac{A \alpha z}{\beta}} .
$$

This gives

$$
\begin{aligned}
& 608 \alpha A(2 m-1) z^{2} \\
& \quad+12 z(\alpha A(37-59 m)+20 \alpha D+8) \\
& \quad-9(3 \alpha A(m-1)-4 \alpha D+8)=0, \\
& 9 A(\alpha-\alpha m)+76 \alpha A(5 m-1) z^{2} \\
& \quad+3 \alpha A(31-75 m) z+18 \alpha D+24 z(5 \alpha D+2) \\
& \quad-36=0
\end{aligned}
$$

from (22) and (24), respectively, and

$$
\begin{aligned}
v= & 1-\frac{\alpha}{1440}\left\{\alpha A ^ { 2 } \left[m^{2}\left(-4636 z^{2}+2880 z+135\right)\right.\right. \\
& +m\left(3496 z^{2}-3180 z-270\right)-76 z^{2}+300 z \\
& +135]-60 A[-9 \alpha D \\
& +m(9 \alpha D+50 \alpha D z+20 z-18)-2 z(5 \alpha D+2) \\
& +18]+540 D(\alpha D-4)\}
\end{aligned}
$$


from (21). Equations (28) and (29) are, in general, not equivalent for arbitrary $z$. However, in both cases when $z=z_{1}$ or $z=z_{2}$, required by (23) and (25), they express the same condition. This shows that (22) and (24) are equivalent, just as (23) and (25), so (21)-(25) supply only three independent conditions.

Solving (28) for $D$ yields

$$
D=\frac{-27 \alpha A+27 \alpha A m-1216 \alpha A m z^{2}+708 \alpha A m z+608 \alpha A z^{2}-444 \alpha A z-96 z+72}{12 \alpha(20 z+3)}
$$

Substitution of $B$ and $D$ into (30) gives a long formula for the wave's velocity:

$$
\begin{aligned}
v= & -\left\{\alpha ^ { 2 } A ^ { 2 } \left[24320(m(1607 m-1382)+323) z^{4}\right.\right. \\
& -3840(m(7703 m-7368)+1791) z^{3} \\
& +576(m(5919 m-7324)+2044) z^{2} \\
& \left.+4320(m-1)(42 m-19) z+1215(m-1)^{2}\right] \\
& +69120 \alpha A(2 m-1) z(2 z(76 z-43)-3) \\
& -2880(8 z(34 z+75)+45)\} \\
& \times\left(\left[5760(20 z+3)^{2}\right]\right)^{-1}
\end{aligned}
$$

the explicit form of which will be presented in the next section, after specifying the branch of $z$ and taking into account conditions implied by periodicity and volume conservation.

\section{Periodicity and Volume Conservation Conditions}

Denote

$$
\begin{aligned}
u(B y, m)= & \operatorname{dn}^{2}(B y, m) \\
& \pm \sqrt{m} \mathrm{cn}(B y, m) \operatorname{dn}(B y, m) .
\end{aligned}
$$

The periodicity condition implies

$$
u(B L, m)=u(0, m) \Longrightarrow L=\frac{4 K(m)}{B},
$$

where $K(m)$ is the complete elliptic integral of the first kind. Note that the wavelength $L$ given by (34) is two times greater than that for a single $\mathrm{cn}^{2}$ periodic solution [21].

Then volume conservation requires

$$
\int_{0}^{L} \eta_{ \pm}(B y, m) d y=\frac{A}{2} \int_{0}^{L} u(B y, m) d y+D L=0 .
$$

Volume conservation means that elevated and depressed (with respect to the mean level) volumes are the same over the period of the wave.

From properties of elliptic functions

$$
\begin{aligned}
\int_{0}^{L} u(B y, m) d y & =\frac{E(\operatorname{am}(4 K(m) \mid m) \mid m)}{B} \\
& =\frac{4 E(m)}{B},
\end{aligned}
$$

where $E(\Theta \mid m)$ is the elliptic integral of the second kind, $\operatorname{am}(x \mid m)$ is the Jacobi elliptic function amplitude, and $E(m)$ is the complete elliptic integral of the second kind. Then from (35)-(36) one obtains $D$ in the form

$$
D=-\frac{A E(m)}{2 K(m)}
$$

In order to obtain explicit expressions for coefficients $A, B, D, v$ one has to specify $z$. Choose the positive root first.

Case $1\left(z=z_{2}=(43+\sqrt{2305}) / 152 \approx 0.59875\right)$. With this choice $A>0$ and the cnoidal wave has crests elevation larger than troughs depression with respect to still water level.

Substitution of $z_{2}$ into (28) (or equivalently (29)) supplies another relation between $A$ and $D$, giving

$$
D=-\frac{12(-51+\sqrt{2305})+37(5-m) \alpha A}{444 \alpha} .
$$

Equating (37) with (38) one obtains $(E K(m)$ is given by $(16))$

$$
A=\frac{12(51-\sqrt{2305})}{37 \alpha E K(m)} .
$$

With this $A$

$$
\begin{aligned}
& B=\sqrt{\frac{12(\sqrt{2305}-14)}{703 \beta E K(m)},} \\
& D=-\frac{6(51-\sqrt{2305})}{37 \alpha E K(m)} \frac{E(m)}{K(m)} .
\end{aligned}
$$

Velocity formula (32) simplifies to

$$
\begin{aligned}
v= & \frac{9439-69 \sqrt{2305}}{5476} \\
& +\left(\frac{7811 \sqrt{2305}-377197}{520220}\right) \frac{\left(m^{2}+14 m+1\right)}{[E K(m)]^{2}} \\
\approx & 1.11875-0.00420523 \frac{\left(m^{2}+14 m+1\right)}{[E K(m)]^{2}} .
\end{aligned}
$$

In general, as stated in previous papers [15, 21, 22], the $\mathrm{KdV} 2$ equation imposes one more condition on coefficients of solutions than $\mathrm{KdV}$. Let us discuss obtained results in 


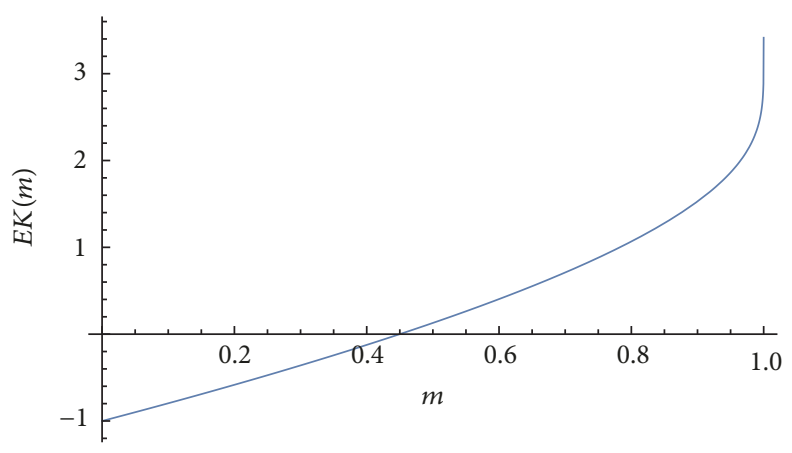

Figure 1: Function $E K(m)$ given by (16).

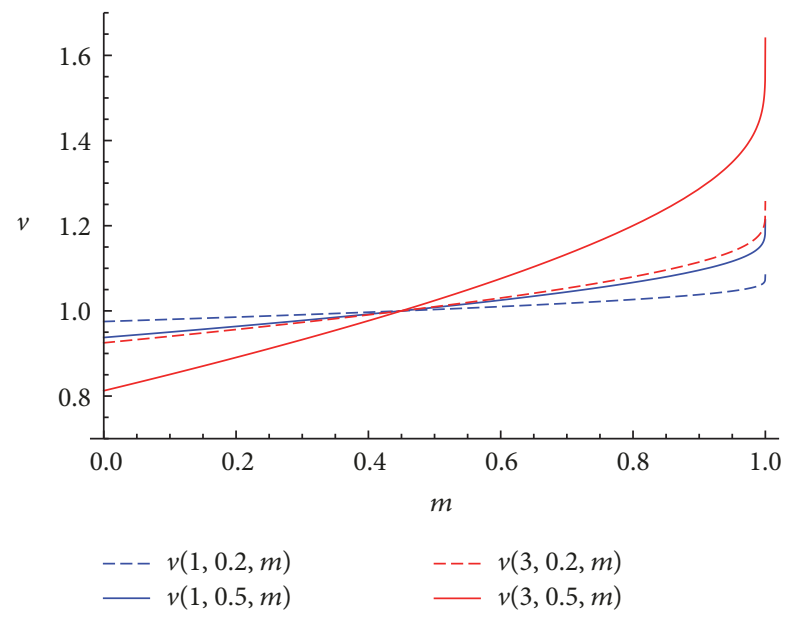

Figure 2: Velocity $v(A, \alpha, m)$ (15) of solution (4) for different $A$ and $\alpha$.

more detail. Coefficients $A, B, D, v$ are related to the function $E K(m)$. This function is plotted in Figure 1.

It is clear that for real-valued $B$ the amplitude $A$ has to be positive, and therefore $m$ must be greater than $\approx 0.45$. Since $B$ depends on $m$ this condition imposes a restriction on wavenumbers. The $m$-dependence of coefficients $A, B, D$ and velocity $v$ (39)-(42) are displayed in Figures 3-6. It is worth noting that $v$ given by (42) contrary to KdV case (15) depends only on $m$.

For $m$ close to 1 the wave height, that is, the difference between the crest's and trough's level, is almost equal to $A / 2$. It is clear from Figure 3 that the wave height is reasonably small for $m$ close to 1 .

Case $2\left(z=z_{1}=(43-\sqrt{2305}) / 152 \approx-0.0329633\right)$.

$$
\begin{aligned}
& A=\frac{12(\sqrt{2305}+51)}{37 \alpha E K(m)}, \\
& B=\sqrt{-\frac{12(\sqrt{2305}+14)}{703 \beta E K(m)},} \\
& D=-\frac{6(\sqrt{2305}+51)}{37 \alpha E K(m)} \frac{E(m)}{K(m)} .
\end{aligned}
$$

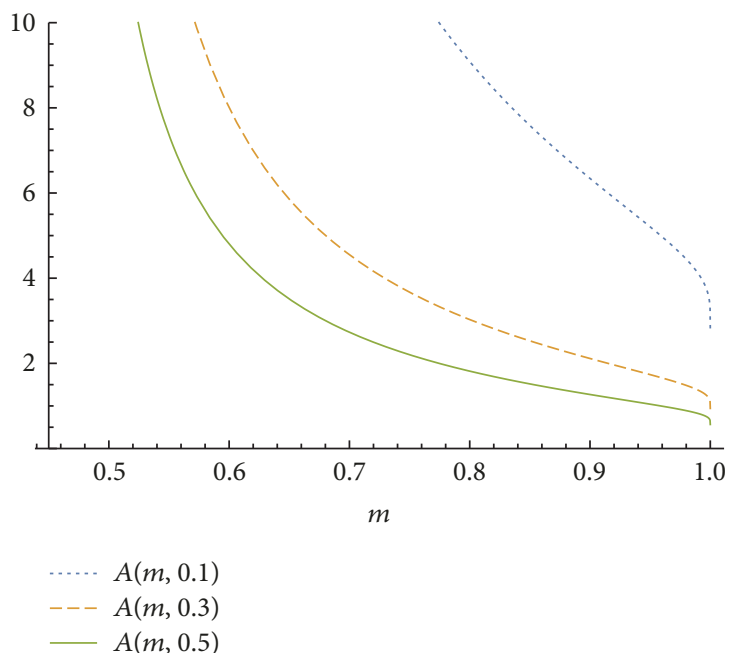

Figure 3: Amplitude $A(m, \alpha)$ (39) of solution (4) as function of $m$ for $\alpha=0.1,0.3,0.5$.

Velocity formula (32) simplifies to

$$
\begin{aligned}
v= & -\frac{9439+69 \sqrt{2305}}{5476} \\
& +\left(\frac{7811 \sqrt{2305}+377197}{520220}\right) \frac{\left(m^{2}+14 m+1\right)}{[E K(m)]^{2}} \\
\approx & -2.32866+1.44594 \frac{\left(m^{2}+14 m+1\right)}{[E K(m)]^{2}} .
\end{aligned}
$$

In this case $B$ is real-valued when $E K(m)$ is negative, that is, for $m$ less that $\approx 0.45$ (see, e.g., Figure 1 ). But this means that $A$ is negative; that is, the cnoidal wave has an inverted shape (crests down, troughs up). Figures 7-10 illustrate examples of $m$-dependence of coefficients $A, B, D, v$ for $m<0.449$.

\section{Examples and Numerical Simulations}

Table 1 contains several examples of coefficients $A, B, D, v$ and the wavelength $L$ of superposition solutions to KdV2 for some particular values of $\alpha, \beta$, and $m$ for the branch $z=z_{2}$. 


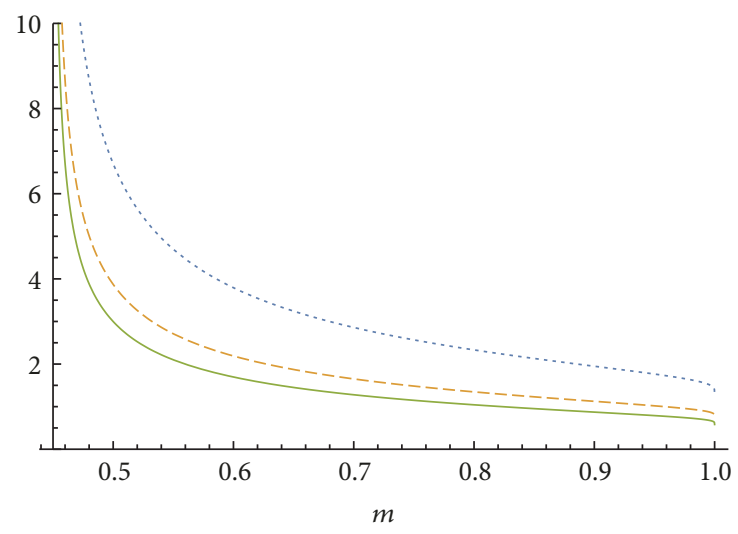

$\ldots B(m, 0.1)$
$--B(m, 0.3)$
$-\quad B(m, 0.5)$

Figure 4: Coefficient $B(m, \beta)$ (40) of solution (4) as function of $m$ for $\beta=0.1,0.3,0.5$.

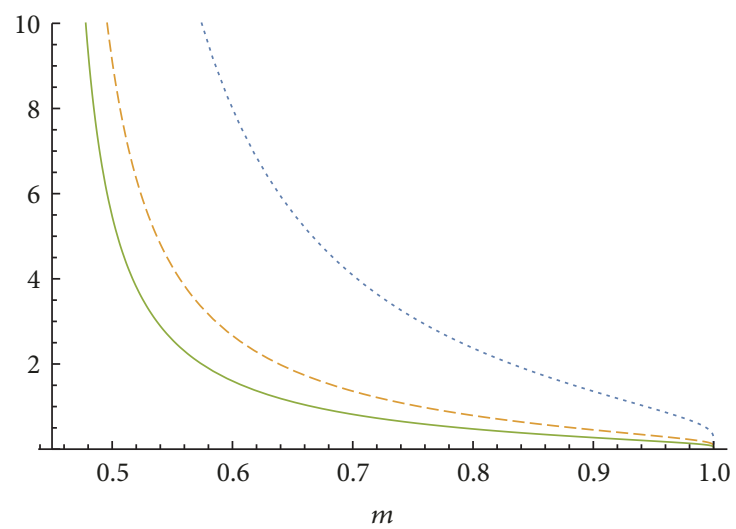

$\cdots \cdot d(m, 0.1)$

$--d(m, 0.3)$

$-d(m, 0.5)$

Figure 5: Coefficient $D(m, \alpha)$ (41) of solution (4) as function of $m$ for $\alpha=0.1,0.3,0.5$.

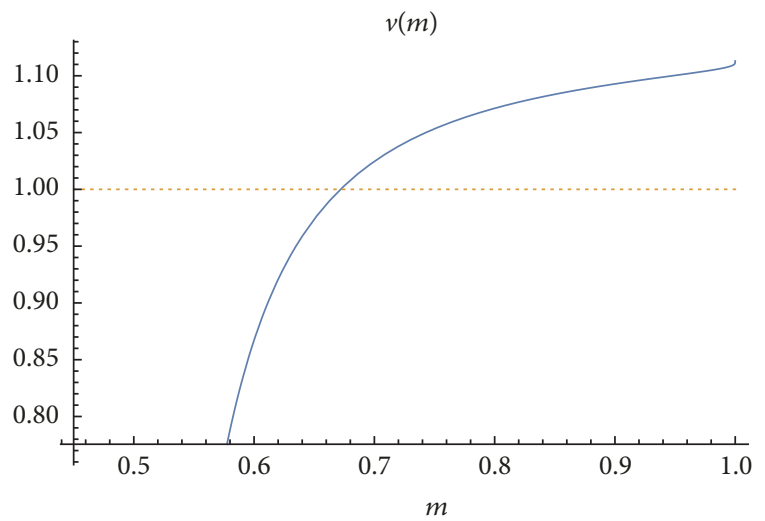

Figure 6: Velocity $v(m)$ (42) of solution (4) as function of $m$.

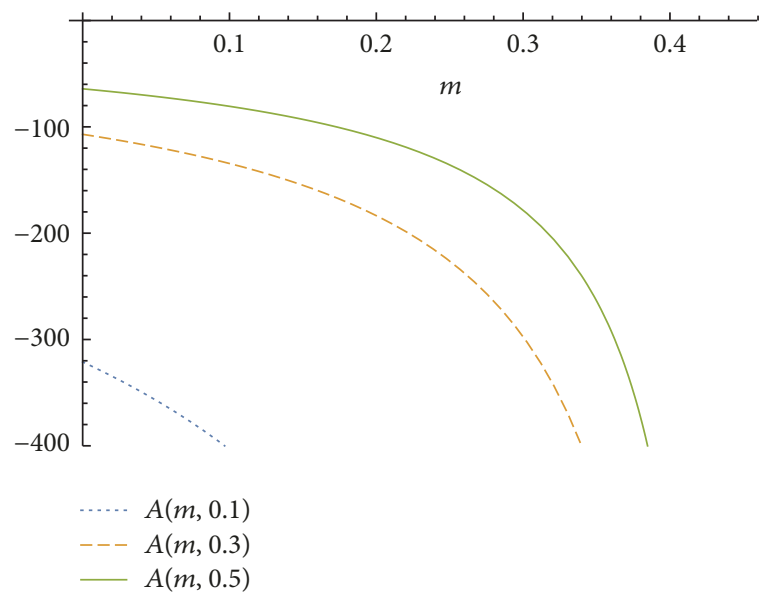

Figure 7: Amplitude $A(m, \alpha)$ (43) of solution (4) as function of $m$ for $\alpha=0.1,0.3,0.5$.

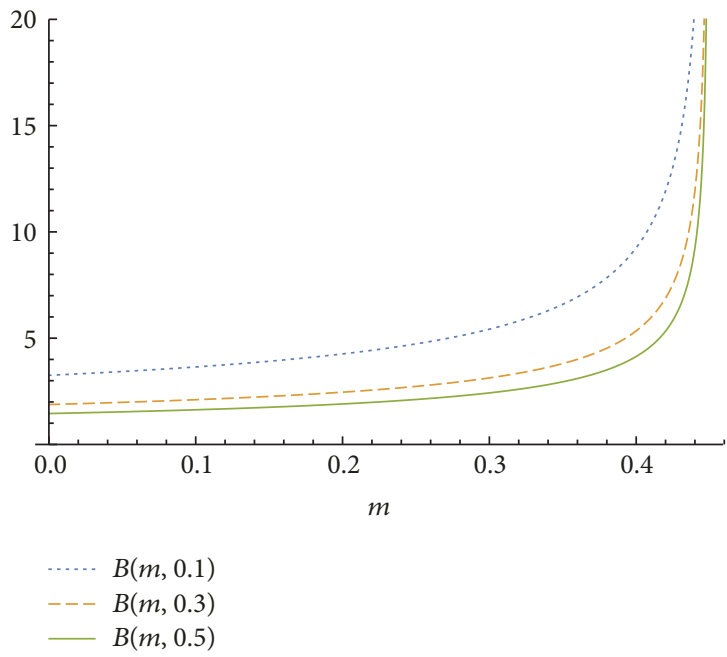

FIgURE 8: Coefficient $B(m, \beta)$ (44) of solution (4) as function of $m$ for $\beta=0.1,0.3,0.5$.

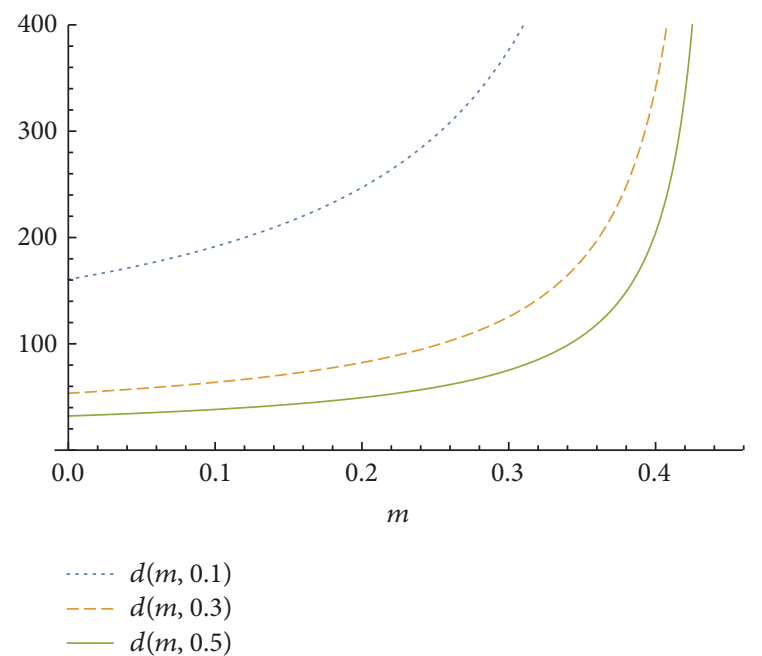

FIgURE 9: Coefficient $D(m, \alpha)$ (45) of solution (4) as function of $m$ for $\alpha=0.1,0.3,0.5$. 


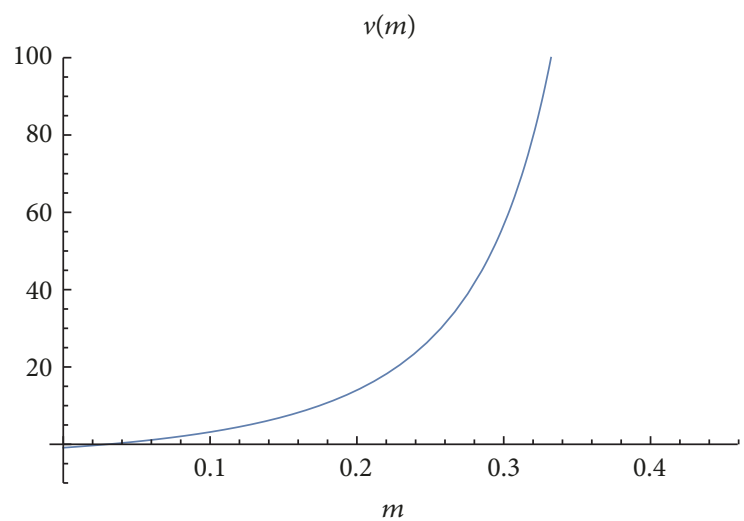

Figure 10: Velocity $v(m)$ (46) of solution (4) as function of $m$.

TABLE 1: Examples of values of $A, B, D, v$, and $L$ for $z=z_{2}$ case.

\begin{tabular}{cccccccc}
\hline$\alpha$ & $\beta$ & $m$ & $A$ & $B$ & $D$ & $v$ & $L$ \\
\hline 0.10 & 0.10 & 0.99 & 4.108 & 1.5683 & -0.5646 & 1.107 & 9.426 \\
0.30 & 0.30 & 0.99 & 1.369 & 0.9054 & -0.1882 & 1.107 & 16.33 \\
0.50 & 0.50 & 0.99 & 0.822 & 0.7013 & -0.1129 & 1.107 & 21.08 \\
0.30 & 0.30 & 0.80 & 3.028 & 1.3465 & -0.7904 & 1.071 & 6.706 \\
0.50 & 0.50 & 0.80 & 1.817 & 1.0430 & -0.4743 & 1.071 & 8.657 \\
\hline
\end{tabular}

TABLE 2: Examples of values of $A, B, D, v$, and $L$ for $z=z_{1}$ case.

\begin{tabular}{cccccccc}
\hline$\alpha$ & $\beta$ & $m$ & $A$ & $B$ & $D$ & $v$ & $L$ \\
\hline 0.30 & 0.30 & 0.10 & -134.5 & 2.105 & 63.83 & 3.170 & 3.064 \\
0.50 & 0.50 & 0.05 & -71.44 & 1.535 & 34.82 & 0.717 & 4.147 \\
\hline
\end{tabular}

Figure 11 displays a comparison of a solution of KdV2 to solution of $\mathrm{KdV}$. For comparison, parameters $\alpha, \beta$ of the equations were chosen to be $\alpha=\beta=0.3$. Compared are waves corresponding to $m=0.99$. Coefficients $A, B, D, v$ of KdV2 solution are given in the second raw of Table 1. For comparison $\mathrm{KdV}$ solution is chosen with the same $A$ but $B, D, v$ are given by (11), (14), and (15), respectively.

Table 2 gives two examples of coefficients $A, B, D, v$ and the wavelength $L$ of superposition solutions to KdV2 for some particular values of $\alpha, \beta$ and small $m$ for the branch $z=z_{1}$.

In Figure 12 profiles of the solution to KdV2 for the cases $\alpha, \beta=0.5$ and $m=0.05$ are displayed for $t=0, T / 3,2 T / 3, T$. In this case we obtain an inverted cnoidal shape, with crest depression equal to -8.885 and trough elevation equal to 7.088 .

In the cases $\alpha, \beta=0.3$ and $m=0.1$ the corresponding values of crest and trough are -24.67 and 17.85 , respectively.

For $m$ close to 1 the wave height is much smaller than the coefficient $A$ and there exists an interval of small $m$ where the wave height is physically relevant.

Numerical calculations of the time evolution of superposition solutions performed with the finite difference code as used in previous papers $[14,15,21,22]$ confirm the analytic results. Numerical evolution of any of the presented solution shows their uniform motion with perfectly preserved shapes. The case corresponding to $z=z_{2}$ branch, with parameters

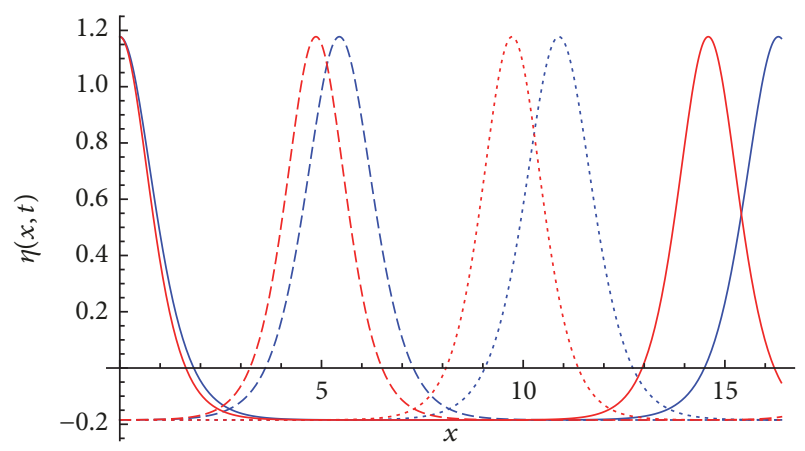

FIGURE 11: Profiles of the KdV2 (blue lines) and KdV solutions (red lines) for $\alpha=\beta=0.3$ and $m=0.99$. Solid lines correspond to $t=0, T$, dashed lines to $t=T / 3$, and dotted lines to $t=2 T / 3$, respectively ( $T$ is the wave period).

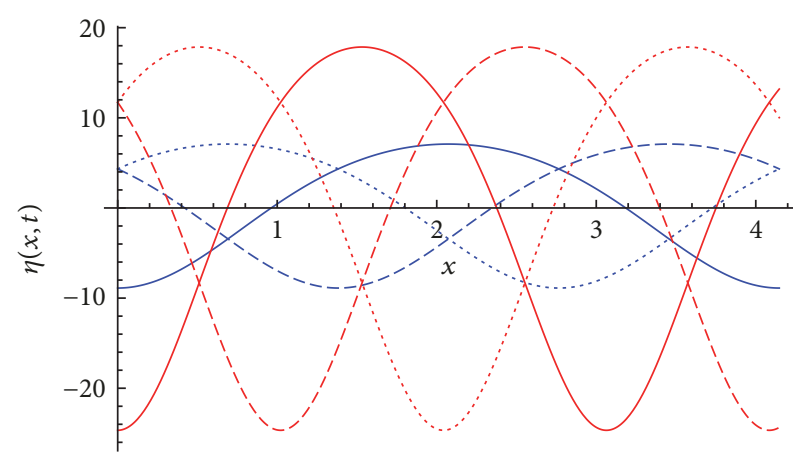

FIgURE 12: Profiles of the KdV2 solutions for $\alpha=\beta=0.5$ and $m=$ 0.05 (blue lines) and for $\alpha=\beta=0.3$ and $m=0.1$ (red lines). Solid lines correspond to $t=0, T$, dashed lines to $t=T / 3$, and dotted lines to $t=2 T / 3$, respectively ( $T$ is the wave period corresponding to the case).

listed in the second raw of Table 1, is illustrated in Figure 13. This is the same wave as that displayed in Figure 11 (blue lines).

The case corresponding to $z=z_{1}$ branch, with parameters listed in the first raw of Table 2, is illustrated in Figure 14. This is the same wave as that displayed in Figure 12 (blue lines).

Remark 1. From periodicity of the Jacobi elliptic functions it follows that

$$
\eta_{+}(x, t)=\eta_{-}\left(x \pm \frac{L}{2}, t\right) .
$$

This means that both $\eta_{+}(x, t)$ and $\eta_{-}(x, t)$ represent the same wave but shifted by half of the wavelength with respect to one another.

\section{Conclusions}

From the studies on the KdV2 equation presented in this paper and in $[15,21]$ one can draw the following conclusions.

(i) There exist several classes of exact solutions to KdV2 which have the same form as the corresponding 


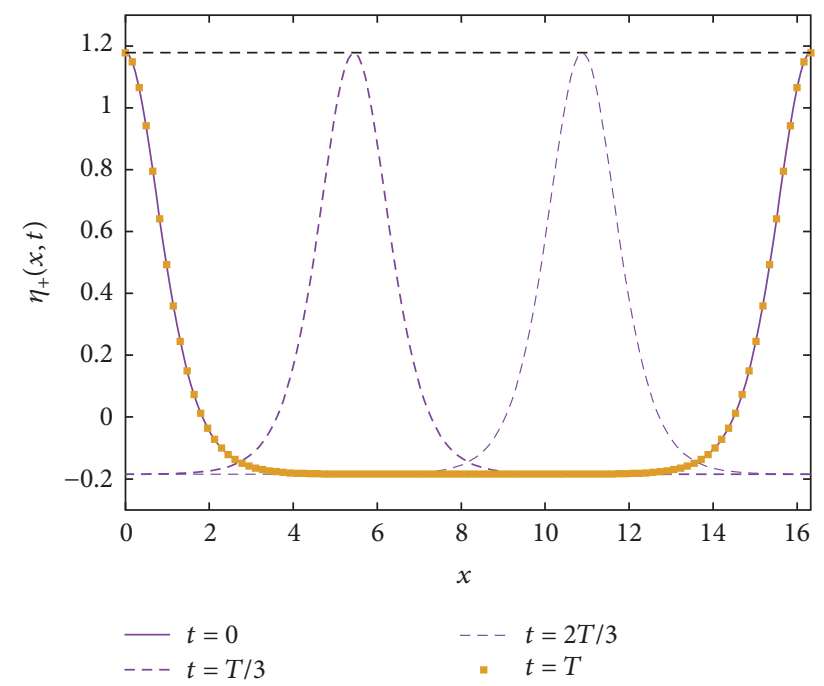

FIgURE 13: Time evolution of the superposition solution $\eta_{+}(x, t)$ for $\alpha=\beta=0.3$ and $m=0.99$ obtained in numerical simulations. Profiles of the wave at $t=0, T / 3,2 T / 3, T$ are shown, where $T$ is the period. Thexinterval is equal to the wavelength.

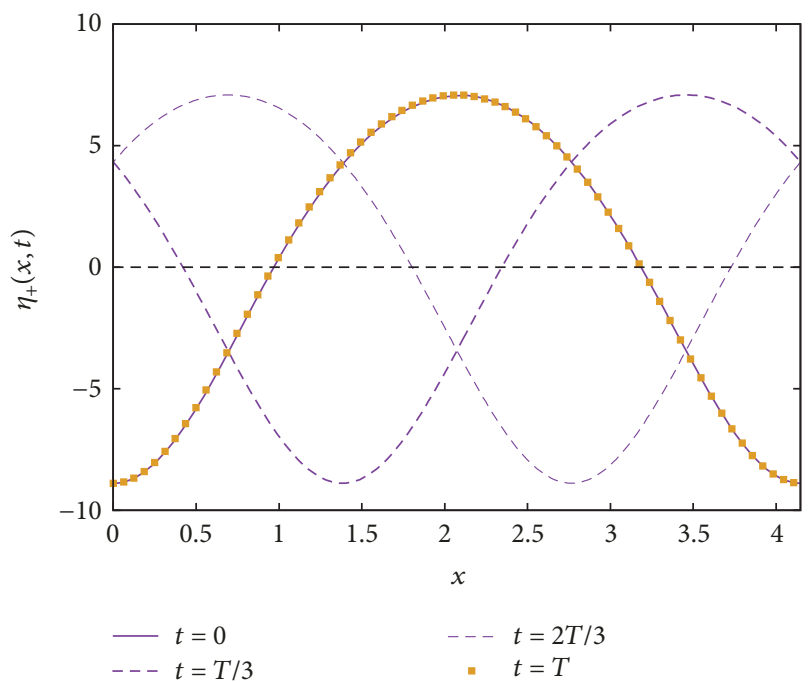

FIgURE 14: Time evolution of the superposition solution $\eta_{+}(x, t)$ for $\alpha=\beta=0.3$ and $m=0.05$ obtained in numerical simulations. Profiles of the wave at $t=0, T / 3,2 T / 3, T$ are shown, where $T$ is the period. Thexinterval is equal to the wavelength.

solutions to KdV but with slightly different coefficients. These are solitary waves of the form $A \mathrm{cn}^{2}$ [15], cnoidal waves $A \mathrm{cn}^{2}+D$ [21], and periodic waves in form (5), that is, $(A / 2)\left[\mathrm{dn}^{2} \pm \sqrt{m} \mathrm{cndn}\right]+D$, studied in the present paper.

(ii) KdV2 imposes one more condition on coefficients of the exact solutions than KdV.

(iii) Periodic solutions for KdV2 can appear in two forms. The first form, $A \mathrm{cn}^{2}+D$, is, as pointed out in [21], physically relevant in two narrow intervals of $m$, one close to $m=0$ and the other close to $m=1$. The

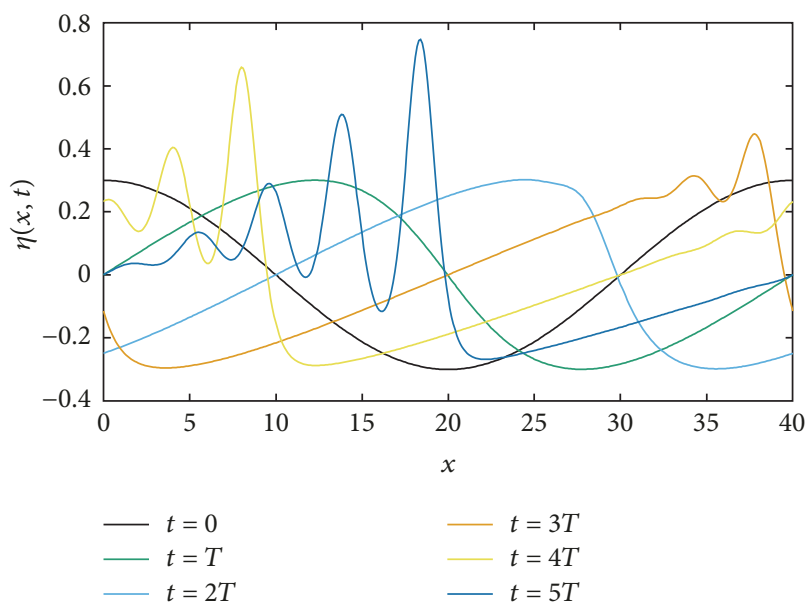

FIGURE 15: Emergence of soliton trains according to KdV from initial cosine wave. $A=0.3, T=50$.

second form, given by (5), gives physically relevant periodic solutions in similar intervals. However, for $m$ close to 1 superposition solution (5) forms a wave similar to $A \mathrm{cn}^{2}+D$, whereas for small $m$ this wave has inverted cnoidal shape.

(iv) All the above-mentioned solutions to KdV2 have the same function form as the corresponding $\mathrm{KdV}$ solutions but with slightly different coefficients.

$\mathrm{KdV}$, besides having single solitonic and periodic solutions, possesses also multisoliton solutions. The question of whether exact multisoliton solutions for KdV2 exist is still open. However, numerical simulations presented in the Appendix, in line with the Zabusky-Kruskal numerical experiment [8], suggest such a possibility. A conjecture that multisoliton solutions to KdV2 might exist in the same form as $\mathrm{KdV}$ multisoliton solutions, but with altered coefficients, will be studied soon.

\section{Appendix}

\section{Do Multisoliton Solutions to KdV2 Exist?}

For KdV there exist multisoliton solutions which can be obtained, for example, using the inverse scattering method $[7,9]$ or the Hirota method [24]. The fact that KdV2 is nonintegrable would seem to exclude the existence of multisoliton solutions to KdV2. On the other hand, numerical simulations demonstrate that for some initial conditions a train of KdV2 solitons, almost the same as that of KdV solitons, emerges from the cosine wave as in Zabusky and Kruskal [8] numerical simulation. We describe such numerical simulation; see Figures 15 and 16.

Initial conditions for both simulations were chosen as hump $\eta(x, 0)=A \cos ((\pi / 40)(x+20))$ for $0 \leq x \leq 40$ and $\eta(x, 0)=0$ for $x>40$ moving to the right. Then such a wave was evolved by a finite difference method code developed in $[14,15,25]$. There is a surprising similarity of trains of solitons obtained in evolution with KdV and KdV2. This behaviour 


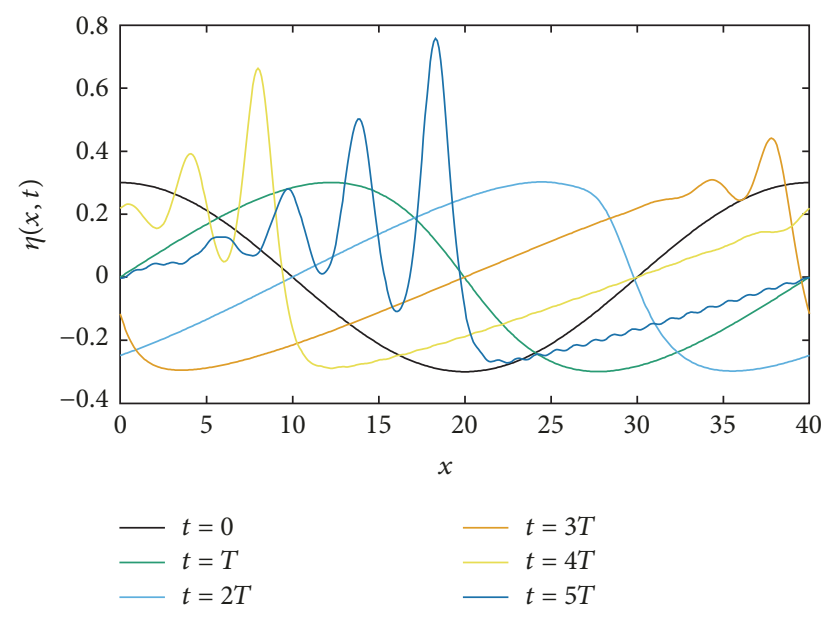

FIGURE 16: Emergence of soliton trains according to KdV2 from initial cosine wave. $A=0.3, T=50$.

might suggest the possible existence of multisoliton KdV2 solutions.

In a multisoliton solution of $\mathrm{KdV}$ each soliton has a different amplitude. Otherwise these amplitudes are arbitrary. If multisoliton solutions to KdV2 exist we would expect some restrictions on these amplitudes.

\section{Conflicts of Interest}

The authors declare that they have no conflicts of interest.

\section{References}

[1] G. G. Stokes, "On the theory of oscillatory waves. Camb. Trans," 1847.

[2] J. V. Boussinesq, "Théorie générale des mouvements qui sont propagés dans un canal rectangulaire horizontal," Comptes rendus de l'Académie des Sciences, vol. 73, pp. 256-260, 1871.

[3] D. J. Korteweg and G. de Vries, "XLI. On the change of form of long waves advancing in a rectangular canal, and on a new type of long stationary waves," Philosophical Magazine, vol. 39, no. 240, pp. 422-443, 1895.

[4] P. G. Drazin and R. S. Johnson, Solitons: An Introduction, Cambridge University Press, Cambridge, UK, 1st edition, 1989.

[5] E. Infeld and G. Rowlands, Nonlinear Waves, Solitons and Chaos, Cambridge University Press, Cambridge, UK, 1990.

[6] M. Remoissenet, Waves called solitons, Springer, Berlin, Germany, 1994.

[7] C. S. Gardner, J. M. Greene, M. D. Kruskal, and R. M. Miura, "Method for solving the Korteweg-deVries equation," Physical Review Letters, vol. 19, pp. 1095-1097, 1987.

[8] N. J. Zabusky and M. D. Kruskal, "Interaction of "solitons" in a collisionless plasma and the recurrence of initial states," Physical Review Letters, vol. 15, no. 6, pp. 240-243, 1965.

[9] M. J. Ablowitz and P. A. Clarkson, Solitons, Nonlinear Evolution Equations and Inverse Scattering, Cambridge University Press, New York, NY, USA, 1991.

[10] G. B. Whitham, Linear and Nonlinear Waves, Wiley, New York, NY, USA, 1974.
[11] M. Dingemans, Water wave propagation over uneven bottoms, World Scientific, World Scientific, Singapore, Singapore, 1997.

[12] T. R. Marchant and N. F. Smyth, "The extended Korteweg-de Vries equation and the resonant flow of a fluid over topography," Journal of Fluid Mechanics, vol. 221, pp. 263-287, 1990.

[13] G. I. Burde and A. Sergyeyev, "Ordering of two small parameters in the shallow water wave problem," Journal of Physics A: Mathematical and Theoretical, vol. 46, no. 7, Article ID 075501, 2013.

[14] A. Karczewska, P. Rozmej, and Ł. Rutkowski, "A new nonlinear equation in the shallow water wave problem," Physica Scripta, vol. 89, no. 5, Article ID 054026, 2014.

[15] A. Karczewska, P. Rozmej, and E. Infeld, "Shallow-water soliton dynamics beyond the Korteweg-de Vries equation," Physical Review E: Statistical, Nonlinear, and Soft Matter Physics, vol. 90, no. 1, Article ID 012907, 2014.

[16] G. Rowlands, P. Rozmej, E. Infeld, and A. Karczewska, "Single soliton solution to the extended $\mathrm{KdV}$ equation over uneven depth," The European Physical Journal E, vol. 40, no. 11, pp. 15, 2017.

[17] A. Karczewska, P. Rozmej, E. Infeld, and G. Rowlands, "Adiabatic invariants of the extended KdV equation," Physics Letters A, vol. 381, no. 4, pp. 270-275, 2017.

[18] A. Khare and A. Saxena, "Linear superposition for a class of nonlinear equations," Physics Letters A, vol. 377, no. 39, pp. 27612765, 2013.

[19] A. Khare and A. Saxena, "Superposition of elliptic functions as solutions for a large number of nonlinear equations," Journal of Mathematical Physics, vol. 55, no. 3, 032701, 25 pages, 2014.

[20] A. Khare and A. Saxena, "Periodic and hyperbolic soliton solutions of a number of nonlocal nonlinear equations," Journal of Mathematical Physics, vol. 56, no. 3, Article ID 1.4914335, 2015.

[21] E. Infeld, A. Karczewska, G. Rowlands, and P. Rozmej, "Solutions to the extended KdV equation for water surface waves," https://arxiv.org/abs/1612.03847.

[22] P. Rozmej, A. Karczewska, and E. Infeld, "Superposition solutions to the extended KdV equation for water surface waves," Nonlinear Dynamics, vol. 91, no. 2, pp. 1085-1093, 2018.

[23] B. Abraham-Shrauner, "Exact solutions of nonlinear partial differential equations," Discrete and Continuous Dynamical Systems - Series S, vol. 11, no. 4, pp. 577-582, 2018.

[24] R. Hirota, "The Bäcklund and inverse scattering transform of the K-dV equation with nonuniformities," Journal of the Physical Society of Japan, vol. 46, no. 5, pp. 1681-1682, 1979.

[25] A. Karczewska, P. Rozmej, and E. Infeld, "Energy invariant for shallow-water waves and the Korteweg-de Vries equation: Doubts about the invariance of energy," Physical Review E: Statistical, Nonlinear, and Soft Matter Physics, vol. 92, no. 5, Article ID 053202, 2015. 


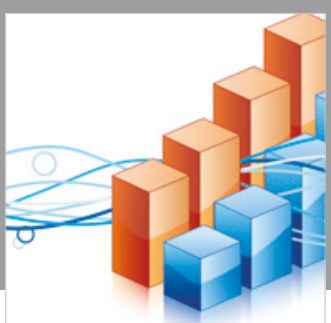

Advances in

Operations Research

\section{-n-m}
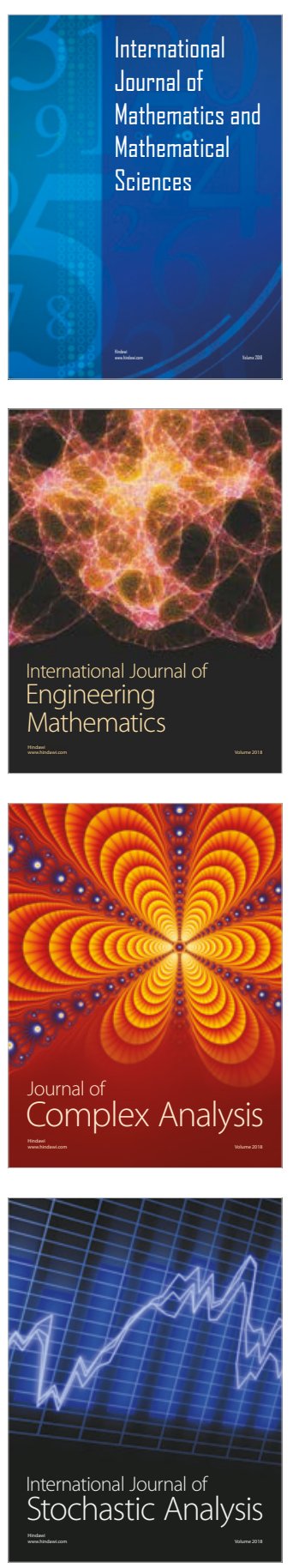
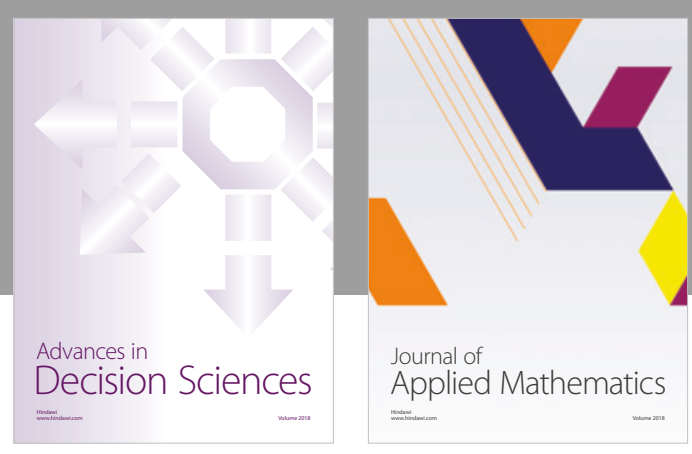

Journal of

Applied Mathematics
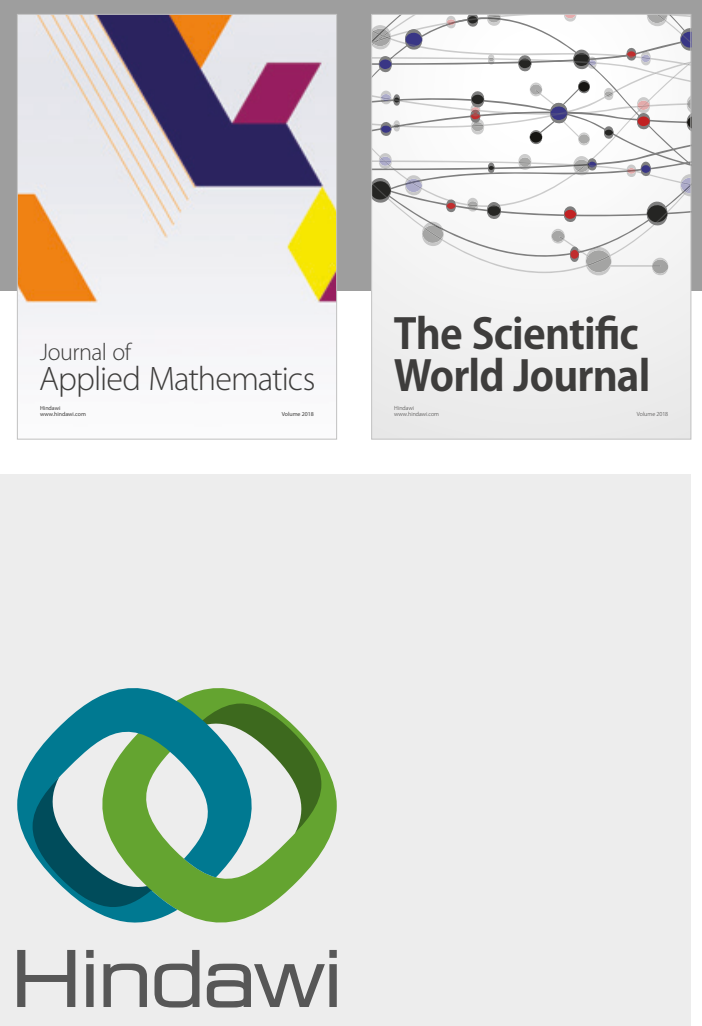

Submit your manuscripts at

www.hindawi.com

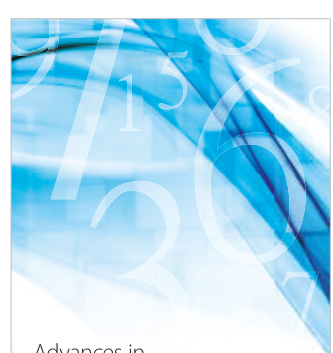

Advances in
Numerical Analysis
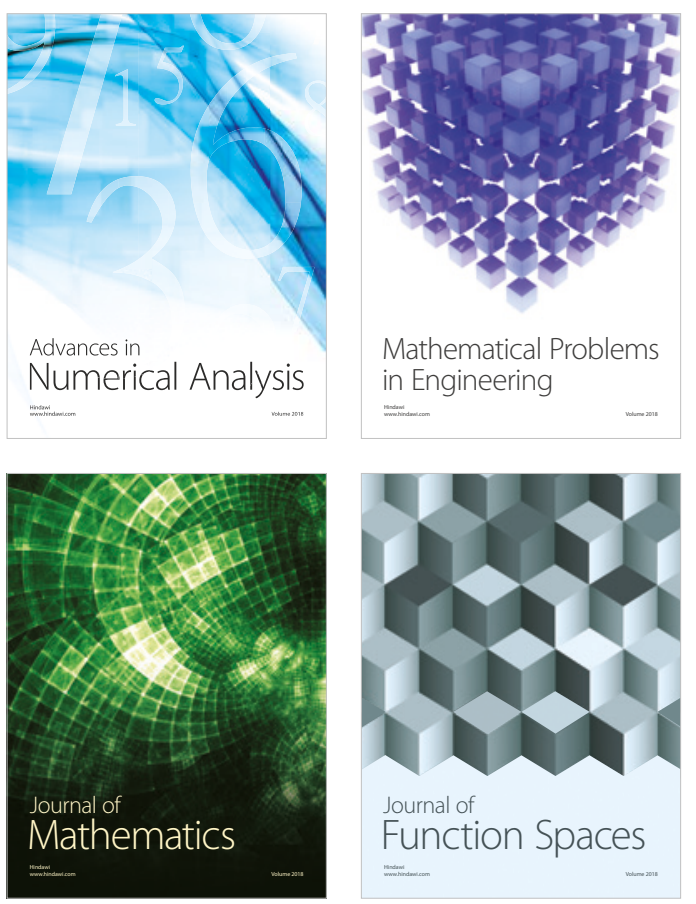

Mathematical Problems in Engineering

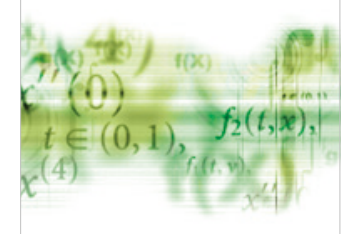

International Journal of

Differential Equations

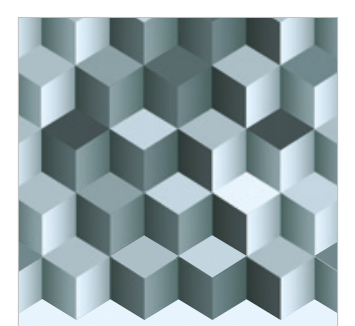

Journal of

Function Spaces

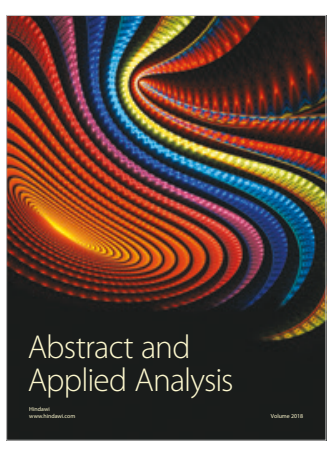

The Scientific

World Journal

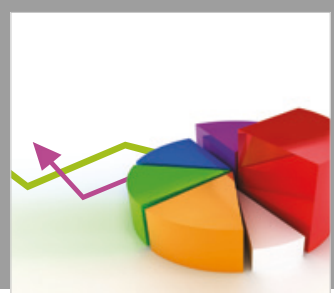

Journal of

Probability and Statistics
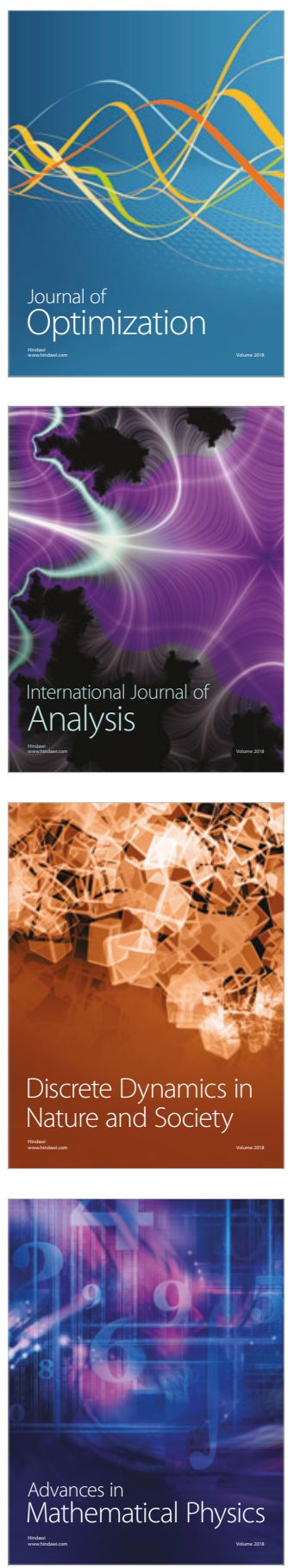